\title{
Diagnostic and Prognostic Value of SHOX2 and SEPT9 DNA Methylation and Cytology in Benign, Paramalignant and Malignant Pleural Effusions
}

\author{
Dimo Dietrich ${ }^{1 * 9}$, Maria Jung ${ }^{19}$, Svenja Puetzer ${ }^{1}$, Annette Leisse ${ }^{1}$, Emily Eva Holmes ${ }^{1}$, Sebastian Meller ${ }^{1}$, \\ Barbara Uhl', Philipp Schatz ${ }^{2}$, Claudia Ivascu ${ }^{3}$, Glen Kristiansen ${ }^{1}$
}

1 University Hospital Bonn (UKB), Institute of Pathology, Bonn, Germany, 2 Metanomics Health GmbH, Berlin, Germany, 3 Roche Pharma AG, Hematology/Oncology, Grenzach-Wyhlen, Germany

\begin{abstract}
Pleural effusions (PE) are a common clinical problem. The discrimination between benign (BPE), malignant (MPE) and paramalignant (PPE) pleural effusions is highly important to ensure appropriate patient treatment. Today, cytology is the gold standard for diagnosing malignant pleural effusions. However, its sensitivity is limited due to the sometimes low abundance of tumor cells and the challenging assessment of cell morphology in cytological samples. This study aimed to develop and validate a diagnostic test, which allows for the highly specific detection of malignant cells in pleural effusions based on the DNA methylation biomarkers SHOX2 and SEPT9. A quantitative real-time PCR assay was developed which enabled the accurate and sensitive detection of SHOX2 and SEPT9 in PEs. Cytological and DNA methylation analyses were conducted in a case control study comprised of PEs from 114 patients (58 cases, 56 controls). Cytological analysis as well as SHOX2 and SEPT9 methylation resulted in $100 \%$ specificity. $21 \%$ of the cases were cytologically positive and $26 \%$ were SHOX2 or SEPT9 methylation positive. The combined analysis of cytology and DNA methylation resulted in an increase of $71 \%$ positively classified PEs from cancer patients as compared to cytological analysis alone. The absolute sensitivity of cytology and DNA methylation was not determinable due to the lack of an appropriate gold standard diagnostic for distinguishing between MPEs and PPEs. Therefore, it was unclear which PEs from cancer patients were malignant (containing tumor cells) and which PEs were paramalignant and resulted from benign conditions in cancer patients, respectively. Furthermore, DNA methylation analysis in PEs allowed the prognosis of the overall survival in cancer patients (Kaplan-Meier analysis, log rank test, $\mathrm{p}=0.02($ SHOX2), $\mathrm{p}=0.02$ (SEPT9)). The developed test may be used as a diagnostic and prognostic adjunct to existing clinical and cytopathological investigations in patients with PEs of unclear etiology.
\end{abstract}

Citation: Dietrich D, Jung M, Puetzer S, Leisse A, Holmes EE, et al. (2013) Diagnostic and Prognostic Value of SHOX2 and SEPT9 DNA Methylation and Cytology in Benign, Paramalignant and Malignant Pleural Effusions. PLoS ONE 8(12): e84225. doi:10.1371/journal.pone.0084225

Editor: Goli Samimi, Kinghorn Cancer Centre, Garvan Institute of Medical Research, Australia

Received July 30, 2013; Accepted November 20, 2013; Published December 27, 2013

Copyright: (C) 2013 Dietrich et al. This is an open-access article distributed under the terms of the Creative Commons Attribution License, which permits unrestricted use, distribution, and reproduction in any medium, provided the original author and source are credited.

Funding: The authors have no support or funding to report.

Competing Interests: Claudia Ivascu and Philipp Schatz are employed at Metanomics Health GmbH and Roche Pharma AG, respectively, whose companies funded time on this study. Dimo Dietrich, Claudia Ivascu and Philipp Schatz have been employees and are stockholders of Epigenomics AG, a company that aims to commercialize the DNA methylation markers SEPT9 and SHOX2. Dimo Dietrich and Philipp Schatz are coinventors and own patents on methylation biomarkers and related technologies. Patents: "A Method for Amplification of Nucleic Acids" (WO2006113770), "A Method for the Carry-over Protection in DNA Amplification Systems Targeting Methylation Analysis Achieved by a Modified Pre-treatment of nucleic Acids" (WO2006040187). These patents are commercially exploited by Epigenomics AG. Dimo Dietrich and Philipp Schatz receive inventor's compensation from Epigenomics AG. There are no further patents, products in development or marketed products to declare. This does not alter the authors' adherence to all the PLOS ONE policies on sharing data and materials.

* E-mail: dimo.dietrich@gmail.com

9 These authors contributed equally to this work.

\section{Introduction}

Pleural effusions (PEs) represent a common clinical complication. Most commonly, PEs are caused by cardiac failure, pneumonia, pulmonary embolism and malignant neoplasm [1,2]. Clinical problems, which result from PEs, are dyspnea, pleural space infections, pleural empyema, and pleural scar tissues $[3,4,5]$. A therapeutic release of the PE is indicated.

Malignancies are the main cause for PEs [2,6]. 50\% up to $65 \%$ of malignancies causing PEs are lung and breast cancers [7]. Malignant pleural effusions (MPEs) may be the initial manifestation of a cancer or may develop during the advanced phases of a known malignancy. The presence of MPEs in cancer patients is associated with an overall poor prognosis leading to an overall survival of less than one year $[8,9,10]$. Treatment of these patients is always palliative $[11,5]$. However, not all cancer patients with a PE appear to have a MPE, but show paramalignant pleural effusions (PPEs). These MPEs occur since cancer patients frequently develop comorbidities such as heart failure, pneumonia, or pulmonary embolism, each of them possibly causing an effusion [11]. Reliable figures regarding the relative number of PPEs and MPEs cannot be found in the literature due to the lack of an accurate gold standard method to discriminate between PPEs and MPEs. A careful evaluation of the effusion is therefore necessary to establish its etiology and to direct the patients' therapy. The discrimination between MPE and PPE might direct a decision towards curative or palliative treatment [11] and is considered for accurate tumor staging according to the TNM-classification. 
The gold standard for diagnosing MPEs is cytology. While it is a highly specific diagnostic tool with up to $100 \%$ specificity $[12,13]$, its sensitivity is limited. The diagnostic sensitivity of cytological analysis averaged over all tumor entities ranges from $40 \%$ up to $90 \%$ and highly depends on the origin of the primary tumor $[2,11,14,15]$. Only few studies analyze cytology with regard to specific tumor entities and report sensitivities ranging from $18 \%$ for lymphoma to $83 \%$ for ovarian cancer [16].

Furthermore, the sensitivity of cytological studies depends on the volume of the pleural fluid sample, the number of specimens, the type of preparation and the experience of the examiner [12,2]. Nevertheless, it is difficult to discriminate malignant from benign cells by morphology in the pleural fluid due to mesothelial and macrophage abnormalities. Actively dividing mesothelial cells for example can mimic adenocarcinoma.

New diagnostic approaches to increase the sensitivity for MPEs are urgently needed. Molecular biomarkers have the potential for improving the clinical management of cancer patients and patients who are suspected of having cancer. A few studies on biomarkers for the discrimination between MPE and BPE have been published so far. The clinical performance of mesothelin, SMRP, survivin, CEA, CA 15-3, CA 19-9, CYFRA 21-1, NSE, TSA have been tested $[17,18,19]$ but the sensitivity did not exceed $35 \%$ at $100 \%$ specificity [19].

DNA methylation is a valuable source for cancer biomarkers. Methylation of the cytosine within the $\mathrm{CpG}$ dinucleotide context plays a key role in fundamental biological processes and human diseases and aberrant DNA methylation is a hallmark of human cancers $[20,21,22,23]$. DNA itself is a chemically robust molecule and DNA methylation is a stable mark. Therefore, DNA methylation biomarkers represent an ideal target for use in clinical routine. However, until now, only one study reported the utility of methylation biomarkers for the diagnosis of MPEs [13].

SHOX2 and SEPT9 are among the most validated DNA methylation markers reported so far. Both biomarkers are already in use in diagnostic tests for lung and colorectal cancer, respectively. Aberrant DNA methylation of SHOX2 is a hallmark of lung cancer tumors and correlates to an amplification of the respective locus 3q25.3 [24,25]. Methylation of the SHOX2 gene locus in bronchial fluid aspirated during bronchoscopy is a validated biomarker in patients with suspected lung cancer and allowed for accurate detection of malignant lung disease even in patients with a negative cytopathological result and no visible tumor in bronchoscopy [26,27]. Furthermore, SHOX2 DNA methylation in plasma is a sensitive and specific biomarker for detecting lung cancer. Sensitivity was particularly high for small cell lung cancer and squamous cell carcinoma [28]. A CE-marked in vitro diagnostic (IVD) test to aid pathologists in the diagnosis of lung cancer based on the SHOX2 DNA methylation biomarker is commercially available in Europe [27].

SEPT9 DNA methylation has been reported to be a powerful biomarker for colorectal cancer [29,30,31]. SEPT9 DNA methylation occurs early on during carcinogenesis and can already be found in precancerous lesions, i.e. adenomas [32]. Thus, the analysis of SEPT9 DNA methylation in blood plasma is a promising test for colorectal cancer screening [29,30,31]. The SEPT9 DNA biomarker was recently validated in a large observational prospective colorectal cancer screening trial (PRESEPT, ClinicalTrials.gov Identifier: NCT00855348, http:// clinicaltrials.gov/ct2/show/NCT00855348) involving nearly 8,000 asymptomatic subjects scheduled to have a colonoscopy [33].

It is well acknowledged that DNA methylation biomarkers are usually not specifically methylated in only a certain tumor entity
[34]. SHOX2 for example is methylated in the different histological subtypes of lung cancer $[24,26,27,28]$. SEPT9 is methylated in colorectal adenocarcinoma and frequently in head and neck squamous cell carcinomas [35]. Hence, it is likely that SHOX2 and SEPT9 are methylated in several different malignancies and represent promising pan cancer biomarkers in clinical questions where the discrimination between malignant and benign disease irrespective of any specificity regarding the origin of a malignant tumor is desired.

The goal of this study was to test the potential of the SHOX2 and SEPT9 DNA methylation biomarkers to improve the sensitivity for detecting malignant cells in PEs and to allow for an accurate prognosis in these patients. In combination with cytology such an assay might significantly improve the clinical management of patients with $\mathrm{PE}$ and may be used as a diagnostic adjunct to existing clinical and cytopathological investigations.

\section{Materials and Methods}

\section{Ethics Statement}

The study has been approved by the Institutional Review Board (IRB) at the University Hospital of Bonn. Informed consent (written) was obtained from all donors or their next of kin.

\section{Patients}

Both cancer patients and patients of the control group donating samples for this study were investigated for suspected cancer at the same clinics at the University Hospital of Bonn between 09/2012 and $07 / 2013$. All PE samples were collected under ultrasound guidance to locate the pleural effusion with a $30 \mathrm{G}$ needle [Becton Dickinson and Company, NJ, USA] under aspiration. PE specimens were fixed with equal volume of Saccomanno's fixative and stored at room temperature. The characteristics of the patients included in this study are shown in Table 1 and Table 2. The presence of malignant disease was confirmed by histology based on biopsy or surgical specimen or by cytology. In one case medical imaging showed a malignant tumor which was not confirmed by histology due to the patient's health condition. Benign conditions were tested by means of microbiological diagnostics, ultrasound, cardiac catheterisation, and x-ray. Only patients who did not have any cancer related history within the last 15 years were considered for the control group. For cytopathological assessment, cytospins or smear preparations from PEs were prepared. The cellular fraction from PE samples containing a high number of cells were formalin-fixed and paraffin-embedded. Sections, cytospins, and smear preparations were analyzed using one or more of the following staining protocols: hematoxylin and eosin (HE), thrombomodulin, TTF1, EpCAM, Periodic Acid Schiff (PAS), Papanicolaou's (PAP), and/or May-GruenwaldGiemsa (MGG).

\section{Sample and Calibrator Preparation}

A calibrator sample (bisulfite-converted artificially methylated human DNA) was prepared as follows: $80 \mu \mathrm{l}$ methylated DNA (100 ng/ $\mu \mathrm{l}$, CpGenome ${ }^{\mathrm{TM}}$ Universal Methylated DNA, Millipore, Billerica, MA, USA), $80 \mu$ l bisulfite reagent (65\% ammonium bisulfite, pH 5.3 [TIB Chemicals, Mannheim, Germany]) and $40 \mu \mathrm{l}$ denaturation reagent $(0.1 \mathrm{~g} / \mathrm{ml}$ hydrochinone [SigmaAldrich, St. Louis, MO, USA] in DEG [diethylene glycol, Sigma-Aldrich, St. Louis, MO, USA]) were mixed and the mixture was incubated for $45 \mathrm{~min}$ at $85^{\circ} \mathrm{C}$ and $1,000 \mathrm{rpm}$ in a thermomixer. The converted DNA was purified by means of Nanosep centrifugal devices (10K, PALL, East Hills, NY, USA) as previously described [36]. The DNA concentration of sperm and 
Table 1. Characteristics of the patient population.

\begin{tabular}{|c|c|c|c|}
\hline & Total & $\begin{array}{l}\text { Cancer } \\
\text { Cases }\end{array}$ & Controls \\
\hline Age & $114(100 \%)$ & $58(100 \%)$ & $56(100 \%)$ \\
\hline$\leq 50$ Years & $14(12 \%)$ & $4(7 \%)$ & $10(18 \%)$ \\
\hline 51-60 Years & 11 (10\%) & $7(12 \%)$ & $4(7 \%)$ \\
\hline$>60$ Years & $89(78 \%)$ & $47(81 \%)$ & $42(75 \%)$ \\
\hline Median Age [Years] & 71.5 & 70 & 73.5 \\
\hline Age Range [Years] & $23-92$ & $30-87$ & $23-92$ \\
\hline \multicolumn{4}{|l|}{ Follow-up } \\
\hline Death & $x$ & $22(38 \%)$ & $x$ \\
\hline Alive & $\mathrm{x}$ & $36(62 \%)$ & $x$ \\
\hline Mean Follow-up [Days] & $\mathrm{x}$ & 62 & $\mathrm{x}$ \\
\hline Median Follow-up [Days] & $\mathrm{x}$ & 36 & $\mathrm{x}$ \\
\hline Range [Days] & $x$ & $0-250$ & $x$ \\
\hline \multicolumn{4}{|l|}{ Gender } \\
\hline Female & $44(39 \%)$ & $25(43 \%)$ & $19(34 \%)$ \\
\hline Male & $70(61 \%)$ & $33(57 \%)$ & 37 (66\%) \\
\hline \multicolumn{4}{|l|}{ Non-Malignant Disease } \\
\hline Heart Diseases & $45(40 \%)$ & $22(38 \%)$ & $23(41 \%)$ \\
\hline $\begin{array}{l}\text { Cardiac Decompensation and } \\
\text { Heart Failure }\end{array}$ & $21(18 \%)$ & $5(9 \%)$ & $16(29 \%)$ \\
\hline Pneumonia & $18(16 \%)$ & $6(10 \%)$ & $12(21 \%)$ \\
\hline Renal Failure & $18(16 \%)$ & $6(10 . \%)$ & $12(21 \%)$ \\
\hline Sepsis & $15(13 \%)$ & $6(10 \%)$ & $9(16 \%)$ \\
\hline Lung Diseases & $9(8 \%)$ & $4(7 \%)$ & $5(9 \%)$ \\
\hline Gastrointestinal Diseases & $8(7 \%)$ & $2(3 \%)$ & $6(11 \%)$ \\
\hline Hepatic Failure & $6(5 \%)$ & $1(2 \%)$ & $5(9 \%)$ \\
\hline Stroke & $6(5 \%)$ & $5(9 \%)$ & $1(2 \%)$ \\
\hline Infectious Diseases & $4(4 \%)$ & $1(2 \%)$ & $3(5 \%)$ \\
\hline Pancytopenia, Anemia & $2(2 \%)$ & $0(0 \%)$ & $2(4 \%)$ \\
\hline $\begin{array}{l}\text { Others (BPH, Hypothyroidism, } \\
\text { etc.) }\end{array}$ & $9(8 \%)$ & $5(9 \%)$ & $4(7 \%)$ \\
\hline \multicolumn{4}{|l|}{ Cytology Result } \\
\hline Positive & 12 (11\%) & $12(21 \%)$ & $0(0 \%)$ \\
\hline Negative & 92 (81\%) & $39(67 \%)$ & 53 (95\%) \\
\hline Suspicious & $10(9 \%)$ & $7(12 \%)$ & $3(6 \%)$ \\
\hline
\end{tabular}

universal methylated DNA was determined by UV spectrophotometry using a Nanodrop ND-1000 spectral photometer (Nanodrop Technologies, Wilmington, DE, USA).

For cut-off validation and analytical performance evaluation, DNA samples with defined methylation levels were prepared by mixing DNA from washed human research sperm (NW Andrology \& Cryobank Inc., Spokane, WA, USA) and CpGenome ${ }^{\text {TM }}$ Universal Methylated DNA. DNA from sperm was extracted using the QIAamp DNA Micro Kit (Qiagen, Hilden, Germany) following the manufacturer's recommendations. The DNA mixtures were bisulfite converted as described above.

The cellular fractions of the PEs were pelleted by centrifugation for $10 \mathrm{~min}$ at $4000 \mathrm{x} \mathrm{g}$. The cellular pellet was washed twice with PBS buffer. For sample lysis $100 \mu$ lysis buffer (50 mM Tris-HCl,
Table 2. Site (organ) specificity of malignant diseases in 58 cancer cases.

\begin{tabular}{ll}
\hline & \\
\hline Organ & No of Patients (\%) \\
\hline All Sites & $58(100 \%)$ \\
\hline Digestive System & $17(29 \%)$ \\
Stomach & $3(5 \%)$ \\
Small Intestine & $1(2 \%)$ \\
Colon & $4(7 \%)$ \\
Anus, Anal Canal, \& Anorectum & $1(2 \%)$ \\
Liver \& Intrahepatic Bile Duct & $7^{*}(12 \%)$ \\
Pancreas & $1(2 \%)$ \\
\hline Respiratory System & $10(17 \%)$ \\
Larynx & $2(3 \%)$ \\
Lung \& Bronchus & $8^{*}(14 \%)$ \\
Bones \& Joints & $1^{*}(2 \%)$ \\
Skin (Excluding Basal \& Squamous) & $1(2 \%)$ \\
Melanoma-skin & $1(2 \%)$ \\
Breast & $11^{*}(19 \%)$ \\
Genital System & $7(12 \%)$ \\
Uterine Cervix & $1^{*}(2 \%)$ \\
Ovary & $5(9 \%)$ \\
Prostate & $1(2 \%)$ \\
Urinary System & $5(9 \%)$ \\
Kidney \& Renal Pelvis & $4(7 \%)$ \\
Ureter \& other Urinary Organs & $1(2 \%)$ \\
Brain \& other Nervous System & $1(2 \%)$ \\
Endocrine System & $2(3 \%)$ \\
Thyroid & $2(3 \%)$ \\
Lymphoma & $5(9 \%)$ \\
Non-Hodgkin Lymphoma & $3^{*}(5 \%)$ \\
Myeloma & $2(3 \%)$ \\
Leukemia & $2(3 \%)$ \\
Acute Myeloid Leukemia & $1^{*}(2 \%)$ \\
\hline Chronic Myeloid Leukemia & $1^{*}(2 \%)$ \\
\hline Other \& Unspecified Primary Sites & $1(2 \%)$ \\
\hline & \\
\hline
\end{tabular}

*one patient with lung, uterine cervix cancer and non-Hodgkin lymphoma, one patient suffering from lung and liver cancer, one patient with breast and bone cancer, and one patient with acute and chronic myeloid leukemia and breast cancer.

doi:10.1371/journal.pone.0084225.t002

pH 8.4, 1 mM EDTA, $0.5 \%$ [v/v] TWEEN $\left.{ }^{\circledR} 20\right)$ and $10 \mu \mathrm{l} 2 \%$ proteinase $\mathrm{K}$ in lysis buffer were added to each cellular pellet. The mixture was incubated overnight at $60^{\circ} \mathrm{C}$ and $1,000 \mathrm{rpm}$ in a thermomixer. $10 \mu \mathrm{l}$ proteinase $\mathrm{K}(2 \%[\mathrm{w} / \mathrm{v}])$ in lysis buffer were added and the mixture was incubated for additional $4 \mathrm{~h}$ at $60^{\circ} \mathrm{C}$ and $1,000 \mathrm{rpm}$ in a thermomixer. $100 \mu \mathrm{l}$ of this mixture were then mixed with $100 \mu \mathrm{l}$ bisulfite reagent and $50 \mu \mathrm{l}$ denaturation buffer and incubated for $45 \mathrm{~min}$ at $85^{\circ} \mathrm{C}$ and $1,000 \mathrm{rpm}$ in a thermomixer. $500 \mu \mathrm{l}$ binding buffer $(50 \%$ ethanol, $50 \%$ guanidiniumthiocyanate solution $(6 \mathrm{M}$ in $0.1 \mathrm{M}$ Tris; $\mathrm{pH} 7.5)$ [AppliChem GmbH, Darmstadt, Germany]) were added to the reaction mixture. The mixture was transferred onto a NucleoSpin Extract II Column (Macherey \& Nagel, Dueren, Germany) and centrifuged at $14,000 \mathrm{x}$ g for $3 \mathrm{~min}$. The bound DNA was washed with $700 \mu \mathrm{l}$ wash buffer $\left(10 \%\right.$ Trizma ${ }^{\circledR} \mathrm{HCl}$ [Sigma-Aldrich, St. 
Louis, MO, USA], $90 \%$ ethanol) followed by centrifugation $(14,000 \times \mathrm{g}, 1 \mathrm{~min})$. The DNA was desulphonated with $700 \mu \mathrm{l}$ alkaline buffer $(250 \mathrm{mM} \mathrm{NaOH}$ [AppliChem $\mathrm{GmbH}$ ], $75 \%$ ethanol). The column was centrifuged at $14,000 \mathrm{xg}$ for $1 \mathrm{~min}$. After this desulphonation, the membrane was washed twice with wash buffer $\left(1^{\text {st }}: 700 \mu \mathrm{l}\right.$ wash buffer, $1 \mathrm{~min}$ at $14,000 \times \mathrm{g} ; 2^{\text {nd }}$ : $500 \mu \mathrm{l}$ wash buffer, $3 \mathrm{~min}$ at $14,000 \mathrm{x} \mathrm{g}$ ). The silica membrane was dried $10 \mathrm{~min}$ at $60^{\circ} \mathrm{C}$ with open lids. Bisulfite converted DNA was eluted with $70 \mu \mathrm{l}$ elution buffer $(1 \%$ Tris-HCl $1 \mathrm{M}$ pH 8.0 [AppliChem $\mathrm{GmbH}$ ]) for $1 \mathrm{~min}$ at $14,000 \mathrm{x} \mathrm{g}$.

\section{Real-time PCR Quantification of SHOX2 and SEPT9 DNA Methylation}

PCR reaction were carried out in $10 \mu \mathrm{l}$ volumes with the following composition: $70 \mathrm{mM}$ Tris-HCl, $\mathrm{pH}$ 8.4, $12 \mathrm{mM} \mathrm{MgCl}_{2}$, $100 \mathrm{mM} \mathrm{KCl}, 8$ \% glycerol, $0.5 \mathrm{mM}$ each dNTP, 2 U FastStart Taq DNA polymerase [Roche Applied Science, Penzberg, Germany], $0.006 \mu \mathrm{l}$ ROX solution. The ROX solution was prepared as previously described [24]. Oligonucleotides (Table S1) for the triplex assay were modified and optimized based on previously published SEPT9, SHOX2 and ACTB assays [24,31,37]. $25 \mathrm{ng}$ template DNA according to UV quantification was used as template DNA per each single PGR reaction.

PCR was performed using an AB 7500 Fast Real-Time PCR System (Life Technologies Corporation, Carlsbad, CA, USA) using the following temperature profile: $20 \mathrm{~min}$ at $95^{\circ} \mathrm{C}$ followed by 50 cycles with $2 \mathrm{~s}$ at $62^{\circ} \mathrm{C}, 45 \mathrm{~s}$ at $56^{\circ} \mathrm{C}$ (each at $100 \%$ ramp rate) and $15 \mathrm{~s}, 95^{\circ} \mathrm{C}$ (at $75 \%$ ramp rate). Thresholds and baselines were set as follows: 0.015 (threshold SHOX2), 0.01 (threshold SEPT9), 0.02 (threshold ACTB), 3-24 (baseline).

The specificity of the assay for bisulfite converted DNA was confirmed using non-converted genomic template DNA.

\section{Data Evaluation and Statistical Analysis}

Each sample and the calibrator was analysed in triplicate. Sample determinations were considered to be valid when the median of the CT-values met the following quality criterion: $C T_{\text {Sample/ } А С T B} \leq 31.5[23], C T_{\text {Sample/SHOX2 }} \leq 35$, or $C T_{\text {Sample/ }}$ $S E P T 9 \leq 40$. Other samples were excluded from analysis due to insufficient DNA yield.

For each sample, a relative methylation value was determined using the $\Delta \Delta \mathrm{CT}$ method adapted for DNA methylation analyses as previously described [24]. In brief, $\Delta \Delta \mathrm{CT}$ values (here exemplarily shown for $S H O X 2$ ) were computed as follows: $\Delta \Delta \mathrm{CT}_{\text {Sample }}=\Delta C T_{\text {Sample }}-\Delta C T_{\text {Calibrator }}$, where $\Delta C T_{\text {Sample }}=$ $C T_{\text {Sample } / A C T B}-C T_{\text {Sample } / S H O X 2}$ and $\Delta C T_{\text {Calibrator }}=C T_{\text {Calibrator } /}$

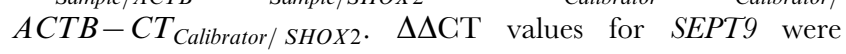
computed accordingly. Percentage methylation was calculated using the following formula: Methylation Sample $=100 \%$. $2^{\Delta \Delta C T_{\text {Sample }}}[24]$.

For $S H O X 2$, a methylation cut-off was assigned for dichotomization of the methylation value. Samples showing a relative SHOX2 methylation level above the cut-off were classified as SHOX2 positive, all others were classified as negative.

Students t-Tests were performed in order to compare SHOX2 and SEPT9 methylation levels of samples of control and cancer patients. Kaplan-Meier analysis was conducted to investigate the overall survival (OS). Overall survival time indicates the time difference between the date of the release of the pleural effusion and the date of death or the last contact, respectively. OS were compared using the log rank test. A correlation between SHOX2 and SEPT9 methylation was tested using a Pearson's correlation. Analysis of variance (ANOVA) was conducted to check for an inter-run variability of test results. All statistical analyses were performed using the SPSS software version 21 (IBM, Armonk, NY, USA). P-values $<0.05$ were considered statistically significant.

\section{Results}

\section{Assay Principle and Analytical Performance Evaluation}

A quantitative real-time PCR triplex assay was developed to accurately and simultaneously assess the methylation level of SHOX2 and SEPT9 DNA in PEs. Methylation-specific PCR (MSP) [38] together with the $\mathrm{TaqMan}^{\mathrm{TM}}$ probe technology for real-time PCR detection were used. Quantification of total DNA content was based on the $\beta$-actin gene $(A C T B)$ in a methylation independent manner. All assays (SHOX2, SEPT9 and ACTB) were performed in a single-tube triplex real-time PCR assay.

In order to investigate the analytical performance of the assay unmethylated DNA was spiked with different amounts of artificially methylated DNA. Both, methylation levels of SEPT9 and $S H O X 2$, were assessed with high precision and accuracy (Figure S1). The assay showed a dynamic range between $0 \%$ and $100 \%$ methylation and was therefore suitable for quantifying methylation levels of SHOX2 and SEPT9.

\section{Clinical Performance}

The developed assay was used in a case control study comprised of PEs from 114 patients (58 cancer patients, 56 control patients with benign diseases). Patient samples yielding insufficient DNA for an accurate methylation analysis were excluded. Therefore, the previously described $A C T B$-CT criterion [27] was combined with predefined exclusion criteria based on SHOX2 and SEPT9 CT values. Since malignant cells in PEs usually represent a minor fraction of the total cells, a low input amount decreases the probability of the presence of DNA from malignant cells for statistical reasons and therefore leading to a false negative result. Out of the 114 analyzed patient samples, 80 yielded sufficient DNA according to the previously described sample exclusion criteria.

As shown in Figure 1, low level methylation of SHOX2 was also found in samples from patients with benign diseases. Therefore, the introduction of a methylation cut-off was required in order to dichotomize the results and to transfer the quantitative result into a qualitative result. Out of 58 cancer patient samples, 12 (21\%) showed detectable SEPT9 methylation and seven $(12 \%)$ showed SHOX2 DNA methylation levels above the cut-off and were defined as methylation positive. The methylation levels of SHOX2 and SEPT9 correlated significantly with each other (Figure 2). 15 out of 58 cancer patient samples (26\%) showed SHOX2 or SEPT9 methylation positivity (Figure 1, Table 3). Positive results in 21 out of 58 cases $(36 \%)$ were obtained by the combination of cytological and DNA methylation analyses. Therefore, the number of positively classified PEs from cancer patients increased by $71 \%$ when combining DNA methylation analysis and cytology as compared to cytological assessment alone. All control patients were methylation negative and showed a negative cytology leading to a specificity of $100 \%$.

Hypermethylation was detected in PEs of patients with different cancer types (Table S2). The highest SHOX2 methylation levels were found in samples from patient with breast (SHOX2: $363 \%$, SEPT9: 0\%), a patient with stomach (SHOX2: 270\%, SEPT9: 22\%) and a patient with lung cancer (SHOX2: 51\%, SEPT9: 16\%). Highest SEPT9 levels were found in PE samples from two patients with stomach cancer (SHOX2: 16\%, 270\%, SEPT9: 65\%, 22\%) and a patient suffering from lung cancer (SHOX2: 51\%, SEPT9: $16 \%)$. 
Table 3. Clinical performance of the DNA methylation biomarkers SHOX2 and SEPT9 and cytology in PEs from 114 patients.

\begin{tabular}{|c|c|c|c|c|c|}
\hline Diagnostic Result & Total Number & Cases & Controls & Positivity & Specificity \\
\hline Cytology + & $12 / 114$ & $12 / 58$ & $0 / 56$ & $21 \%$ & \\
\hline Cytology - & $92 / 114$ & $39 / 58$ & $53 / 56$ & & $100 \%$ \\
\hline Cytology (t) & 10/114 & $7 / 58$ & $3 / 56$ & & \\
\hline SHOX2+ & $7 / 114$ & $7 / 58$ & $0 / 56$ & $12 \%$ & \\
\hline SHOX2 - & 73/114 & $31 / 58$ & $42 / 56$ & & $100 \%$ \\
\hline SHOX2 nd & $34 / 114$ & $20 / 58$ & $14 / 56$ & & \\
\hline SEPT9+ & $12 / 114$ & $12 / 58$ & $0 / 56$ & $21 \%$ & \\
\hline SEPT9 - & $68 / 114$ & $26 / 58$ & $42 / 56$ & & $100 \%$ \\
\hline SEPT9 nd & $34 / 114$ & $20 / 58$ & $14 / 56$ & & \\
\hline SEPT9 or SHOX2 + & $15 / 114$ & $15 / 58$ & $0 / 56$ & $26 \%$ & \\
\hline SEPT9 and SHOX2 -/nd & $99 / 114$ & $43 / 58$ & $56 / 56$ & & $100 \%$ \\
\hline SEPT9 or SHOX 2 or Cytology + & $21 / 114$ & $21 / 58$ & $0 / 56$ & $36 \%$ & \\
\hline SEPT9 and SHOX2 and Cytology $-/(+) /$ nd & $93 / 114$ & $37 / 58$ & $56 / 56$ & & $100 \%$ \\
\hline
\end{tabular}

Positive test results are labelled " + ", cytologically suspicious for malignancy were labelled by " $(+)$ ". Negative test results are labelled by " - ". Invalid test results due to low DNA content are shown as "nd". The results classified as " +$)^{\prime \prime}$ and "nd" were used equivalently to negative test results in the calculation of positivity and specificity. doi:10.1371/journal.pone.0084225.t003

Patients with a MPE are expected to have a poorer outcome as compared to patients with PPE since patients with MPE usually present at more advanced stages. Therefore, a Kaplan-Meier survival analysis of the cancer patients stratified by the $S H O X 2$ and SEPT9 methylation levels was conducted. Figure 3 shows that cancer patients with a SHOX2 or SEPT9 methylation positive PE have a significantly shorter overall survival as compared to cancer patients with a methylation negative PE. A positive cytological result was not a significant predictor for adverse outcome. This finding supports the hypothesis that methylation positivity in PEs from cancer patients allows for the discrimination between malignant and paramalignant PEs.
SHOX2

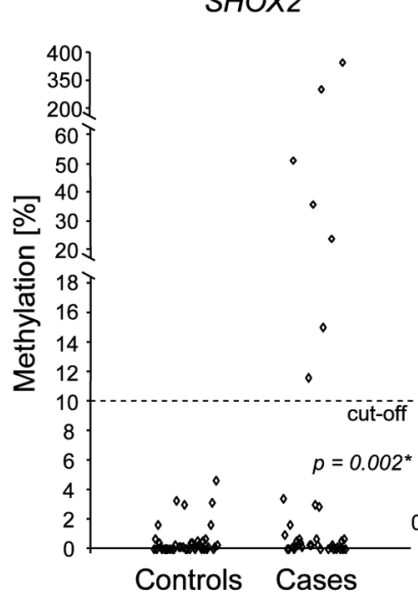

SEPT9

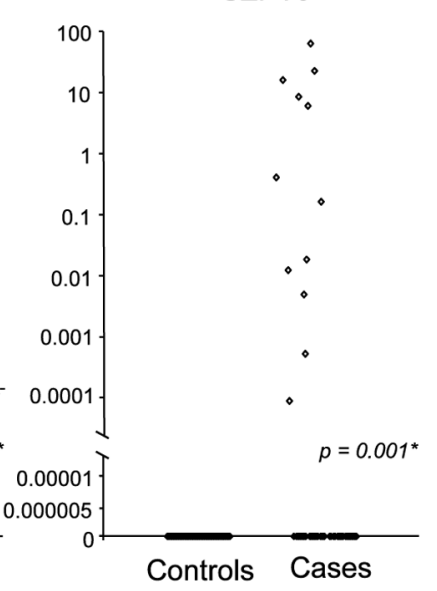

Figure 1. SHOX2 and SEPT9DNA methylation in PEs from cancer patients and patients with benign diseases. Methylation values of SHOX2 and SEPT9 in PEs from patients with cancer (cases) and patients with benign diseases (controls) determined by quantitative real-time $P C R$. The $p$-values refer to a Students t-test. A methylation cut-off was used to classify patient samples as SHOX2 positive (above the cut-off). doi:10.1371/journal.pone.0084225.g001

\section{Cut-off Validation}

The introduction of a methylation cut-off for dichotomization of the SHOX2 methylation levels represents a technological challenge. It needs to be ensured that the accuracy and reproducibility of the assay is sufficiently high to correctly classify patient samples which show a methylation level close to the cut-off. Accordingly, the analytical performance of the assay close to the cut-off was validated. Four PE samples from the clinical performance evaluation study as described above were selected which showed a methylation level close to the cut-off. Two samples showed a methylation level above the cut-off and two below. In addition, defined mixtures of artificially methylated DNA and unmethylated DNA were analyzed. The clinical samples and the DNA mixtures were repeatedly measured in nine repeated measurements. Each measurement was comprised of three single replicates resulting in a total of 27 replicates per samples. The measurements were conducted in three independent runs in order to determine the variability and robustness of the assay. The assay allowed for an accurate discrimination of samples showing a SHOX2 methylation level above and below the cut-off (Figure 4, Table 4). Overall variability of SHOX2 and SEPT9 DNA methylation quantification

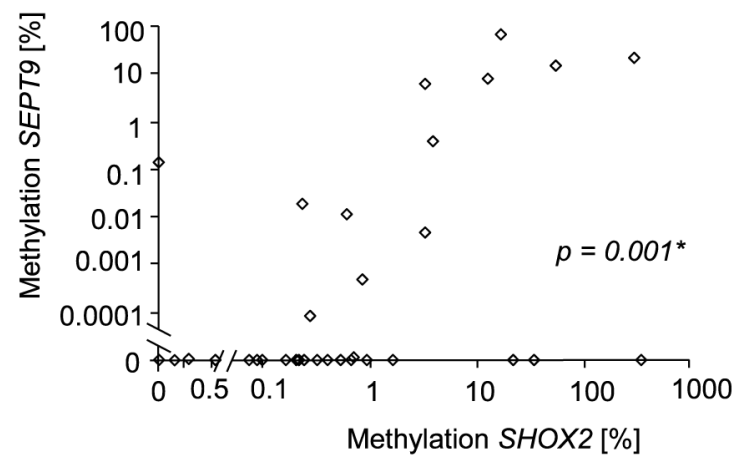

Figure 2. Correlation of SHOX2 and SEPTO DNA methylation in PEs. Correlation of the methylation values of SHOX2 and SEPT9 in PEs from cancer patients. The $p$-value refers to a Pearson's correlation. doi:10.1371/journal.pone.0084225.g002 


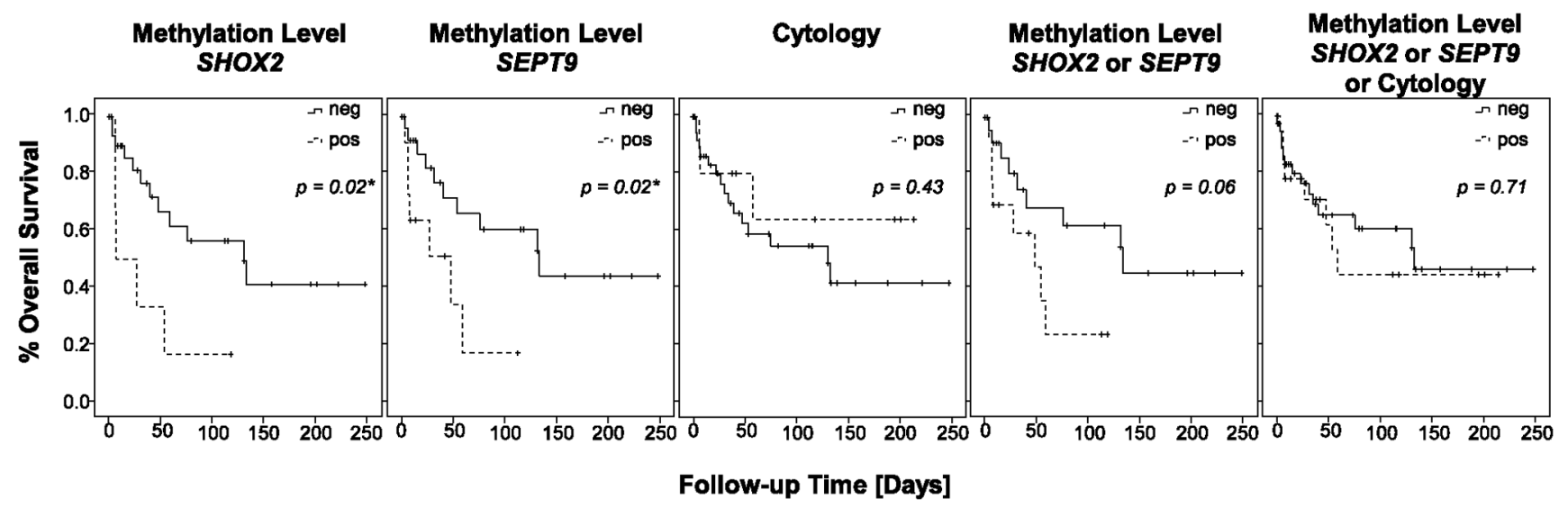

Figure 3. Survival analyses. Kaplan-Meier analysis of overall survival in 58 cancer patients stratified by the cytological diagnosis and the DNA methylation status of SHOX2 and SEPT9 in PEs. The p-values refer to the log rank test. doi:10.1371/journal.pone.0084225.g003

was low. No significant inter-run variability was found, as all ANOVA p-values were greater than 0.05 .

\section{Discussion}

In this study, DNA methylation of the SHOX2 and SEPT9 gene loci were shown to be highly specific biomarkers for the diagnosis of malignant pleural effusions. While the specificity was $100 \%$ for both biomarkers, the apparent overall positivity of $26 \%$ for SHOX2 and SEPT9 represented an improvement as compared to cytology alone. However, due to lack of a highly accurate diagnostic gold standard for the differential diagnosis of PEs, this positivity does not represent the sensitivity of the test. The true

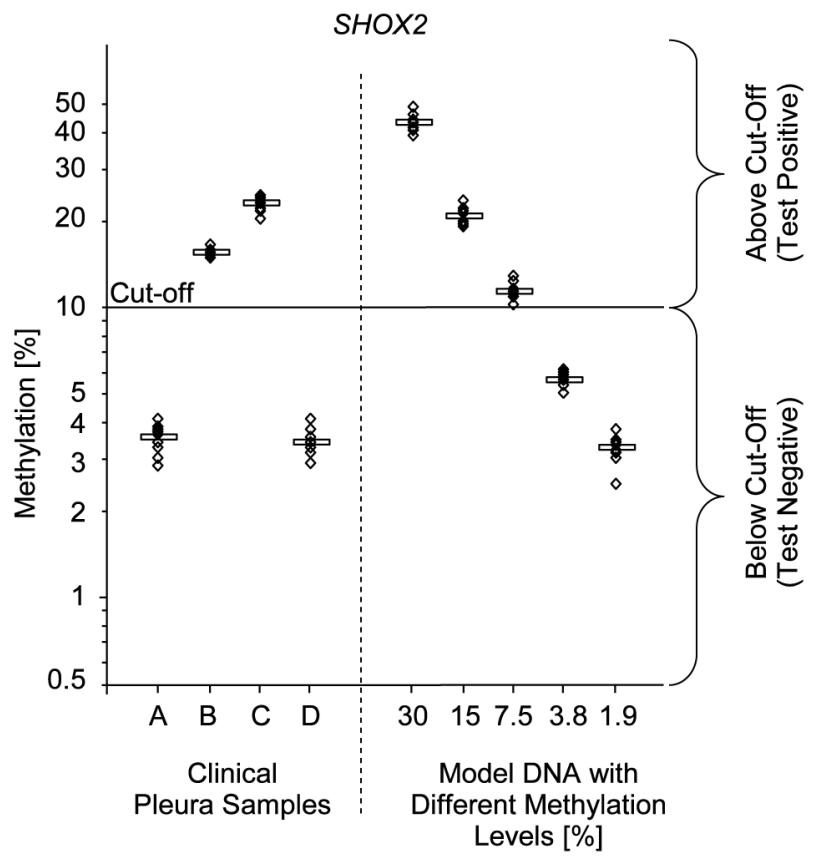

Figure 4. Cut-off validation. Cut-off validation for the SHOX2 diagnostic assay. Model DNA with levels of unmethylated and artificially methylated DNA in addition to clinical samples were measured repeatedly in different runs. Methylation values of each sample and model DNA were measured in nine replicates.

doi:10.1371/journal.pone.0084225.g004 number of MPEs is most likely higher than the observed positivity since not all PEs from cancer patients are in fact MPEs. Cytology for example does not allow the differentiation between false negative MPEs and truly negative PPEs. Therefore, a positivity of $100 \%$ cannot be expected even from a diagnostic test with $100 \%$ sensitivity since its performance is always related to cytology as a gold standard. In this case the positivity can only reach the level of the portion of MPEs in the group of cancer patients since this group is comprised of patients with MPEs and PPEs. The true number of MPEs in cancer patients analyzed in this study is unclear and therefore the exact sensitivity is speculative. However, since the group of cases and the group of controls analyzed in this case control study do not differ significantly regarding the presence of benign diseases, i.e. heart failure and pneumonia, it is likely that a significant number of PEs from cancer patients were in fact paramalignant. After cancer $(27 \%)$, heart failure $(20 \%)$ and pneumonia $(18 \%)$ are the second and third leading cause, respectively, for the development of PEs [2]. In the presented study, heart failure and pneumonia together were present in $19 \%$ of the cancer patients. Therefore, it can be concluded that a significant number of PEs in cancer patients included in this study were in fact paramalignant and caused by benign diseases. Accordingly, the comparison of the calculated sensitivity of cytological analysis with reported cytological sensitivities is not trivial. Other studies classify PEs from cancer patients with negative results in cytology already as BPE if a benign disease was diagnosed which may have caused the PE $[19,39]$. However, it is not evident if this benign disease in fact caused the PE or if the PE was due to a malignancy. Therefore, the reported sensitivity of cytology in these studies is likely to be overestimated.

Furthermore, this study showed that patients with $S H O X 2$ or SEPT9 positive PEs have an adverse overall survival and the respective methylation levels significantly correlate with a worse outcome. These findings indicate that SHOX2 or SEPT9 in fact allowed for the discrimination between PPEs and MPEs. Patients with MPEs are expected to have a worse outcome as compared to patients with PPEs due to the more advanced stage of the disease in patients with MPEs. SHOX2 DNA methylation has been reported to detect lung cancer in bronchial aspirates with a sensitivity of $68-78 \%$ and a specificity of $95-96 \%$ [26,27]. These studies were based on the analysis of the cellular fraction of bronchial lavage and a positive $S H O X 2$ methylation signal was indicative for the presence of malignant cells. From the technological point of view, the sample material was similar to 
Table 4. Validation of cut-off (SHOX2).

\begin{tabular}{|c|c|c|c|c|c|c|c|c|c|}
\hline & \multicolumn{4}{|c|}{ Clinical pleura samples } & \multicolumn{5}{|c|}{ Model DNA with different methylation levels } \\
\hline & A & B & C & D & $30 \%$ & $15 \%$ & $7.5 \%$ & $3.75 \%$ & $1.875 \%$ \\
\hline \multicolumn{10}{|l|}{ SHOX2 } \\
\hline Mean methylation [\%] & 3.55 & 15.54 & 22.99 & 3.45 & 43.63 & 20.84 & 11.44 & 5.59 & 3.27 \\
\hline SD & 0.43 & 0.61 & 1.29 & 0.36 & 3.03 & 1.36 & 0.78 & 0.39 & 0.38 \\
\hline$\% \mathrm{CV}$ & 12.14 & 3.92 & 5.62 & 10.48 & 6.95 & 6.54 & 6.78 & 6.89 & 11.53 \\
\hline Inter-run (p-value) & 0.44 & 0.20 & 0.27 & 0.16 & 0.06 & 0.09 & 0.19 & 0.41 & 0.76 \\
\hline
\end{tabular}

Statistical analysis of repeated measurements of pleural effusion samples and model DNA samples with different methylation levels close to the cut-off. doi:10.1371/journal.pone.0084225.t004

the sample material from PEs and a similar sensitivity might be expected when analyzing PEs.

Besides the unavailability of a gold standard to discriminate between MPE and PPE and therefore to allow for the calculation of the sensitivity, the design of the presented study implied two major limitations. Firstly, the relatively low number of included patients limits the significance regarding sensitivity and specificity of the test. This is particularly important when regarding the determination of the specificity. A high specificity of ideally $100 \%$ is needed for each single biomarker in order to allow for the combination of several biomarkers without a loss of overall specificity. It is likely that the specificity will decrease when analyzing a higher number of control patients, since the normal population variation might be higher than observed here. This might necessitate shifting the cut-off for dichotomization of the SHOX2 signal towards higher methylation, therefore leading to a loss of sensitivity. Methylation of SEPT9 has been found in plasma of a significant number of patients without malignant disease [33]. Therefore, it is likely that control patients with positive SEPT9 signal might be found when analyzing higher numbers of control patients. This would necessitate the implementation of a cut-off for SEPT9 as well, thus potentially reducing the sensitivity. However, the presented and previous studies showed [26,27] that the level of SHOX2 and SEPT9 methylation directly correlated with the likelihood of the presence of malignant cells. Therefore, the careful evaluation of the measured methylation level prior to result dichotomization might allow the clinicians to draw personalized diagnostic conclusions. The second limitation of this study is the lack of a reference opinion for the cytopathological assessment of all patient samples. It is likely that the overall sensitivity of cytology increases with the number of reference cytopathologists assessing the specimens. This could reduce the potential additive value of a biomarker test in adjunct to cytology. However, it should be considered that a clinically useful diagnostic test needs to work in standard clinical routine where the opinion of several experienced cytopathologists can hardly be obtained. DNA methylation based biomarker tests have been shown to be highly robust and reproducible [27] and therefore can smoothly be implemented into clinical routine without the need of highly experienced personnel.

34 out of $114(30 \%)$ patient samples gave invalid results during methylation analysis due to the lack of a sufficient amount of DNA in the sample. A sufficient number of DNA copies in the sample are required in order to distinguish between false and true negative results. The results might be false negative if the cell number equivalent related to the DNA amount is too low and therefore the likelihood of the presence of DNA from malignant cells is limited as well. However, the presented study was conducted using left- over sample material which was obtained after completion of cytopathological routine diagnostics. A sampling procedure which is optimized with regard to the application to a molecular biological test will increase the number of valid results due to the availability of higher cell numbers. A negative or invalid test result does not have an impact on the diagnosis since cytology is still the gold standard and is included in the test. Accordingly, invalid and negative samples would be handled like patients samples with only cytological results available. However, because of the high positive predictive value of the assay, a positive assay result should be considered for further treatment of patients. The high number of invalid results on the other hand led to a decrease of sensitivity of the test since invalid results of cancer patient samples were treated as false negative results. Therefore, an optimized sample preparation is likely to lead to an increase of sensitivity.

Although SHOX2 is a validated biomarker in lung cancer [26,27], its positivity was low in pleural fluid of lung cancer patients $(13 \%)$. This fact might be attributed to low DNA amounts, paramalignant effusions or the high background methylation of SHOX2 in PEs which necessitated the definition of a high cut-off that was higher than in previous studies where plasma or bronchial aspirates were analyzed [26,27,28]. Further studies including larger patient cohorts are needed to address this question.

The clinical performance of SHOX2 and SEPT9 was similar as compared to published studies. However, the sensitivities of the tumor markers CEA, CA 125, CA 15-3, CYFRA 21-1 and mesothelin strongly depend on the tumor entity. The overall sensitivity of each marker analyzed in all cancer entities did not exceed $35 \%$ when considering $100 \%$ specificity [19]. The introduction of cut-offs and the combination of CEA, CA 125, CA 15-3, CYFRA 21-1 lead to an overall sensitivity of 54\% at $100 \%$ specificity. However, the combination of SHOX2 and SEPT9 methylation biomarkers with additional biomarkers should be taken into consideration. Since SHOX2 and SEPT9 seem to be suitable biomarkers in different tumor entities a benefit regarding the overall sensitivity is possible. For example, SEPT9 allowed for the detection of Non-Hodgkin lymphoma in one of three cases. This entity was not detected by the combination of CEA, CA 125, CA 15-3 and CYFRA 21-1 [19].

A comparison of SHOX2 and SEPT9 and the biomarkers NSA, TSA and CA 19-9 is difficult since the latter were only tested on primary malignancies of the bronchus or the pleura [17]. The diagnostic value of soluble mesothelin-related peptide (SMRP) was mainly investigated for the discrimination between mesothelioma and benign effusions [40,41]. Due to its low diagnostic accuracy the use of survivin is not recommended for the discrimination 
between benign and malignant pleural effusions, but it may be used as a predictive or prognostic biomarker [42].

In summary, this study describes SHOX2 and SEPT9 as promising biomarkers for the diagnosis of MPEs and might be used in adjunct to cytological assessment. Furthermore, the methylation biomarkers SHOX2 and SEPT9 might improve the prognostic accuracy. In particular DNA methylation biomarkers are promising candidates for clinical application where a sensitive detection is required. Hypermethylation of SHOX2 has previously been shown to be frequently accompanied by gene amplification leading to apparent methylation levels above 100\% [25]. The high sensitivity of the SHOX2 DNA methylation biomarker in plasma and bronchial aspirates as reported earlier $[26,27,28]$ could be due to a correlation of DNA methylation and locus amplification in malignant cells.

The assay described here may be used as a diagnostic adjunct to existing clinical and cytopathological investigations in patients with a PE and might allow for an improved patient management.

\section{Supporting Information}

Figure S1 Analytical assay performance. Analytical performance of qPCR assay for accurate and sensitive detection of

\section{References}

1. Marel M, Stastny B, Melínová L, Svandová E, Light RW (1995) Diagnosis of pleural effusions. Experience with clinical studies, 1986 to 1990. Chest 107: 1598-1603.

2. Porcel JM, Light RW (2013) Pleural effusions. Dis Mon 59: 29-57.

3. Bhatnagar R, Maskell NA (2013) Treatment of complicated pleural effusions in 2013. Clin Chest Med 34: 47-62.

4. Mitrouska I, Klimathianaki M, Siafakas NM. (2005) Effects of pleural effusion on respiratory function. Can Respir J. 11: 499-503.

5. Heffner JE. (2008) Diagnosis and management of malignant pleural effusions..Respirology.13: 5-20.

6. Leuallen EC, Carr DT. (1955) Pleural effusion; a statistical study of 436 patients. N Engl J Med. 252: 79-83.

7. DiBonito L, Falconieri G, Colautti I, Bonifacio D, Dudine S (1992) The positive pleural effusion. A retrospective study of cytopathologic diagnoses with autopsy confirmation. Acta Cytol 36: 329-332.

8. Heffner JE, Nietert PJ, Barbieri C (2000) Pleural fluid $\mathrm{pH}$ as a predictor of survival for patients with malignant pleural effusions. Chest 117: 79-86.

9. Gonzalez AV, Bezwada V, Beamis JF Jr, Villanueva AG (2010) Lung injury following thoracoscopic talc insufflation: experience of a single North American center. Chest 137: 1375-1381.

10. Roberts ME, Neville E, Berrisford RG, Antunes G, Ali NJ (2010) BTS Pleural Disease Guideline Group. Management of a malignant pleural effusion: British Thoracic Society Pleural Disease Guideline 2010. Thorax 65 Suppl 2: ii32-40.

11. Antony VB, Loddenkemper R, Astoul P, Boutin C, Goldstraw P, et al. (2001) Management of malignant pleural effusions. Eur Respir J 18: 402-419.

12. Woenckhaus M, Grepmeier U, Werner B, Schulz C, Rockmann F et al. (2005) Microsatellite analysis of pleural supernatants could increase sensitivity of pleural fluid cytology. J Mol Diagn 7: 517-524.

13. Brock MV, Hooker CM, Yung R, Guo M, Han Y et al. (2005) Can we improve the cytologic examination of malignant pleural effusions using molecular analysis? Ann Thorac Surg 80: 1241-1247.

14. Jay SJ (1985) Diagnostic procedures for pleural disease. Clin Chest Med 6: 3348.

15. Maskell NA, Butland RJ (2003) BTS guidelines for the investigation of a unilateral pleural effusion in adults. Pleural Diseases Group, Standards of Care Committee, British Thoracic Society. Thorax. 58 Suppl 2: ii8-17.

16. Rodríguez-Panadero F (2008) Medical thoracoscopy. Respiration. 76: 363-372.

17. Alataş F, Alataş O, Metintaş M, Colak O, Harmanci E el al. (2001) Diagnostic value of CEA, CA 15-3, CA 19-9, CYFRA 21-1, NSE and TSA assay in pleural effusions. Lung Cancer 31: 9-16.

18. Liang QL, Shi HZ, Qin XJ, Liang XD, Jiang J, et al. (2008) Diagnostic accuracy of tumour markers for malignant pleural effusion: a meta-analysis. Thorax 63: $35-41$.

19. Porcel JM, Vives M, Esquerda A, Salud A, Pérez B (2004). Use of a panel of tumor markers (carcinoembryonic antigen, cancer antigen 125, carbohydrate antigen 15-3, and cytokeratin 19 fragments) in pleural fluid for the differential diagnosis of benign and malignant effusions. Chest 126: 1757-1763.

20. Jones PA (2012) Functions of DNA methylation: islands, start sites, gene bodies and beyond. Nat Rev Genet 13: 484-492.

21. Baylin SB, Jones PA (2011) A decade of exploring the cancer epigenome biological and translational implications. Nat Rev Cancer 11: 726-734.
SHOX2 and SEPT9 DNA methylation. Shown are means of triplicate measurements.

(TIF)

Table S1 Oligonucleotide specifications. Sequences, labels and final concentrations of the oligonucleotides used in the quantitative real-time PCR for measuring DNA methylation of SHOX2 and SEPT9 in PEs. Genomic localizations of amplicons refer to assembly GRCh37/hg19.

(DOC)

Table S2 Tumor site specific clinical performance. Positivity of the developed assay in PEs from 58 cancer patients with respect to the primary tumor.

(DOC)

\section{Author Contributions}

Conceived and designed the experiments: DD MJ PS CI GK. Performed the experiments: MJ SP AL BU SM. Analyzed the data: DD MJ. Wrote the paper: DD MJ EEH GK.

22. Shen H, Laird PW (2013) Interplay between the cancer genome and epigenome. Cell 153: 38-55.

23. Suvà ML, Riggi N, Bernstein BE (2013) Epigenetic reprogramming in cancer. Science 339: 1567-1570.

24. Dietrich D, Hasinger O, Liebenberg V, Field JK, Kristiansen G et al.(2012) DNA methylation of the homeobox genes PITX2 and SHOX2 predicts outcome in non-small-cell lung cancer patients. Diagn Mol Pathol 21: 93-104.

25. Schneider KU, Dietrich D, Fleischhacker M, Leschber G, Merk J et al. (2011) Correlation of SHOX2 gene amplification and DNA methylation in lung cancer tumors. BMC Cancer. 11: 102.

26. Schmidt B, Liebenberg V, Dietrich D, Schlegel T, Kneip C et al. (2010) SHOX2 DNA methylation is a biomarker for the diagnosis of lung cancer based on bronchial aspirates. BMC Cancer 10:600.

27. Dietrich D, Kneip C, Raji O, Liloglou T, Seegebarth A et al. (2012) Performance evaluation of the DNA methylation biomarker SHOX2 for the aid in diagnosis of lung cancer based on the analysis of bronchial aspirates. Int $\mathrm{J}$ Oncol 40: 825-832.

28. Kneip C, Schmidt B, Seegebarth A, Weickmann S, Fleischhacker M et al. (2011) SHOX2 DNA methylation is a biomarker for the diagnosis of lung cancer in plasma. J Thorac Oncol 6: 1632-1638.

29. Lofton-Day C, Model F, Devos T, Tetzner R, Distler J et al. (2008) DNA methylation biomarkers for blood-based colorectal cancer screening. Clin Chem 54: 414-423.

30. Grützmann R, Molnar B, Pilarsky C, Habermann JK, Schlag PM et al (2008). Sensitive detection of colorectal cancer in peripheral blood by septin 9 DNA methylation assay. PLoS One 3: e3759.

31. deVos T, Tetzner R, Model F, Weiss G, Schuster M, et al, (2009) Pilarsky C, Habermann JK, Fleshner PR, Oubre BM, Day R, Sledziewski AZ, Lofton-Day C. Circulating methylated SEPT9 DNA in plasma is a biomarker for colorectal cancer. Glin Chem 55: 1337-1346.

32. Tänzer M, Balluff B, Distler J, Hale K, Leodolter A et al. (2010) Performance of epigenetic markers SEPT9 and ALX4 in plasma for detection of colorectal precancerous lesions. PLoS One 5: e9061

33. Church TR, Wandell M, Lofton-Day C, Mongin SJ, Burger M et al. (2013) Prospective evaluation of methylated SEPT9 in plasma for detection of asymptomatic colorectal cancer. Gut. doi:10.1136/gutjnl-2012-304149

34. Heichman KA, Warren JD (2012) DNA methylation biomarkers and their utility for solid cancer diagnostics. Clin Chem Lab Med 50: 1707-1721.

35. Bennett KL, Karpenko M, Lin MT, Claus R, Arab K et al. (2008) Frequently methylated tumor suppressor genes in head and neck squamous cell carcinoma. Cancer Res 68: 4494-4499.

36. Kneip C, Schmidt B, Fleischhacker M, Seegebarth A, Lewin J et al. (2009) A novel method for sensitive and specific detection of DNA methylation biomarkers based on DNA restriction during PCR cycling. Biotechniques 47: 737-744.

37. Lewin J. (2011) Methods for preservation of genomic DNA sequence complexity. United States Patent Application 20110027789.

38. Herman JG, Graff JR, Myöhänen S, Nelkin BD, Baylin SB (1996) Methylationspecific PCR: a novel PCR assay for methylation status of CpG islands. Proc Natl Acad Sci USA 93: 9821-9826. 
39. -1, CEA, CA 19-9, CA 15-3, and CA 125 assays in pleural effusions: analysis of 116 cases and review of the literature. Oncologist. 10: 501-507.

40. Pass HI, Wali A, Tang N, Ivanova A, Ivanov S, et al. (2008) Soluble mesothelinrelated peptide level elevation in mesothelioma serum and pleural effusions. Ann Thorac Surg. 85: 265-272.
41. Scherpereel A, Grigoriu B, Conti M, Gey T, Grégoire M, et al. (2006) Soluble mesothelin-related peptides in the diagnosis of malignant pleural mesothelioma. Am J Respir Crit Care Med. 173:1155-1160.

42. Park DS, Hwang KE, Shim H, Kim BR, Choi KH, et al. (2012) Elevated survivin is associated with a poor response to chemotherapy and reduced survival in lung cancer with malignant pleural effusions. Clin Exp Metastasis. 29:83-89. 